Revista de

Contabilidade e

Organizações

www.rco.usp.br
DOI: http://dx.doi.org/10.11606/rco.v11i29.1225503
Journal of

Accounting and

Organizations

www.rco.usp.br

\title{
Análise comparativa de distintas métricas de risco na composição de um fundo de fundos de investimento imobiliário
}

\author{
Lucas Fernando Lovatto ${ }^{\mathrm{a}}$; Daniel Christian Henrique ${ }^{\mathrm{b}}$; Marcus Vinícius Andrade de Lima ${ }^{\mathrm{b}}$ \\ ${ }^{a}$ Banco Regional de Desenvolvimento do Extremo Sul \\ ${ }^{b}$ Universidade Federal de Santa Catarina
}

\section{Informações do Artigo}

Histórico do Artigo

Recebido: 12 de janeiro de 2017

Aceito: 20 de maio de 2017

\section{Palavras-chave:}

Fundos de Investimento Imobiliário.

Conditional Value-at-Risk.

Análise de Investimentos.

Otimização de Carteiras.

\begin{abstract}
Resumo
O propósito central deste estudo é analisar comparativamente os resultados de diferentes métricas de risco na composição de um Fundo de Fundos de Investimento Imobiliário (FIIs). A partir da revisão de literatura, optou-se pela adoção das métricas de risco variância e Conditional Value-at-Risk (CVaR), desenvolvidas nos estudos de Markowitz (1952) e Rockafellar e Uryasev (2000, 2002), respectivamente. O estudo abrangeu uma amostra de 30 FIIs listados em bolsa de valores no âmbito da BMF\&BOVESPA com retornos diários entre julho de 2013 e julho de 2015. Como metodologia, empregou-se modelagem e simulação computacional de carteiras teóricas para minimização do risco não-sistemático, submetendo-as a restrições de acordo com perfis de risco e instruções regulatórias. A principal constatação é de que as diferentes métricas produziram diferentes resultados nos portfólios de menor nível de risco. Nesta situação, o CVaR minimizou perdas mais acentuadas, decorridas de seu foco em risco downside. A métrica de risco variância, por sua vez, subestimou a probabilidade de eventos oriundos de tail risk, sendo sub-ótimo para investidores que possuem como objetivo primário a minimização de risco, mesmo que possivelmente com menor potencial de retorno esperado. Todavia, a partir de certo patamar de risco, ambas as métricas igualam seus resultados. Estes resultados puderam ser verificados em todas as fronteiras eficientes dos fundos analisados.
\end{abstract}

Copyright (C) 2017 FEA-RP/USP. Todos os direitos reservados

\section{INTRODUÇÃO}

Indivíduos, empresas e instituições financeiras enfrentam problemas na alocação de recursos entre as inúmeras alternativas de investimentos no mercado de capitais nos dias atuais. Em consequência, emerge naturalmente a seguinte questão: como maximizar o patrimônio com exposição ao menor risco possível? A gênese do que hoje é chamada de Moderna Teoria do Portfólio (MPT) ou Análise Média-Variância são os estudos de Markowitz $(1952,1959)$, nos quais pela primeira vez foi estudado o comportamento do risco e retorno de conjuntos de ativos ao invés de ativos separados, surgindo conceitos como diversificação e portfólios eficientes.

Recentes estudos desenvolvidos com relação à MPT são relacionados à incorporação de custos de transação, maior precisão na estimação dos parâmetros, novos métodos de diversificação e otimizações multiperíodo (Kolm, Tütüncü, \& Fabozzi, 2014). Entretanto, algumas premissas da MPT começaram a ser questionadas nos anos 90: a variância é a medida de risco mais correta? Todos os retornos de ativos são distribuídos normalmente? A partir desses questionamentos, surge um novo conceito de risco: o downside risk. Nesta metodologia, o alvo de estudo passa a ser as caudas de perdas (ou tails) das distribuições dos retornos. Esses novos estudos também são chamados de Pós-Moderna Teoria do Portfólio (sigla PMPT, em inglês), considerada uma extensão da MPT (Rom \& Ferguson, 1994).

Ambas as metodologias podem ser alocadas na análise de uma nova classe de ativos no mercado de capitais brasileiro, os Fundos de Investimento Imobiliário (FIIs), que têm atraído a atenção de investidores, sobretudo individuais. Embora criados em 1993, somente após uma série de novas regulamentações e incentivos é que o setor cresceu significativamente, no final dos anos 2000. Acompanhando o elevado crescimento da construção civil no período entre 2008 e 2013, a emissão de FIIs cresceu mais de 250\% no mesmo período, passando de 73 para 257 (Associação Brasileira das Entidades dos Mercados Financeiro e de Capitais [ANBIMA], 2014). 
Em decorrência da complexidade que pode gerar a análise deste significativo número de fundos aos investidores, a adoção estratégica dos Fundos de Fundos consolida uma saída viável e equilibrada na confrontação de riscos e retornos. Também são chamados de fundos multigestores, ou seja, são fundos de investimento que investem diretamente em cotas de outros fundos de investimento. Essa estratégia permite que investidores tenham acesso a um portfólio altamente diversificado adquirindo apenas um ativo, não requerendo análises individuais para tomada de decisão quanto à diversificação e composição de seu próprio portfólio de fundos (Strachman \& Bookbinder, 2010). Dentro deste contexto, o objetivo desta pesquisa é analisar de forma comparativa o riscovariância e risco downside em portfólios otimizados de Fundos de FIIs.

\section{REVISÃO TEÓRICA}

\subsection{Diversificação do risco}

Os ativos podem ser classificados dentro de duas simples segmentações: com risco ou livre de risco. Um ativo livre de risco é definido como aquele em que o investidor possui certeza do retorno esperado. Isto posto, seu risco-variância é igual a zero (Reily \& Brown, 2011; Vernimmen, Quiry, Dallocchio, Le Fur, \& Salvi, 2014).

Por outro lado, na análise do risco presente em ativos de risco, constata-se a presença de diversas fontes: riscos operacional, financeiro, de liquidez, de taxa de câmbio, de mercado, de país. Mas uma diferença importante pode ser delineada: os riscos chamados não-sistemáticos (riscos específicos do ativo a ser analisado) são passíveis de diversificação, enquanto que o risco sistemático (risco de mercado) não é possível de ser eliminado pela diversificação. As análises primárias deste fenômeno foram desenvolvidas por Markowitz (1952, 1959) (Bodie, Kane, \& Marcus, 2011; Reilly \& Brown, 2011; Berk, Demarzo, \& Harford, 2012). Situações de variação do risco sistemático e não-sistemático do portfólio mediante a quantidade de ativos alocados são apresentadas na Figura 1.

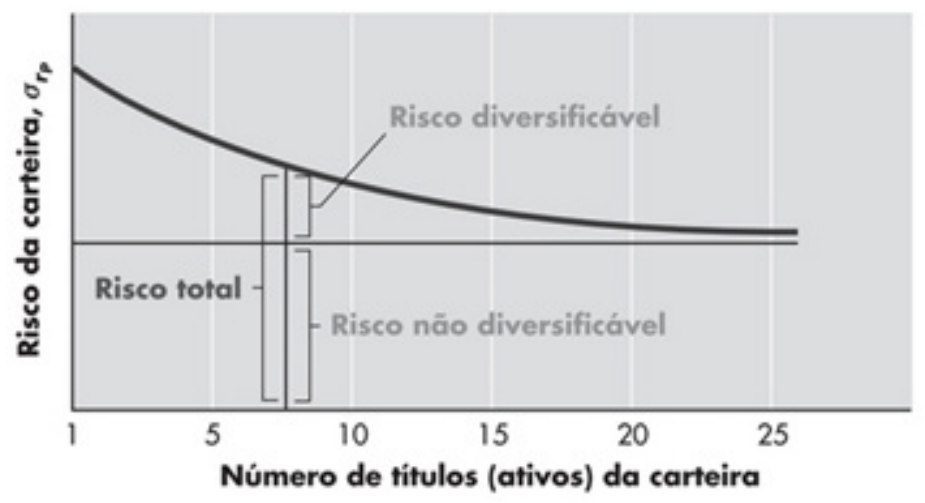

Figura 1. Efeito da diversificação no risco do portfólio Fonte: Gitman (2010)

Assim, portfólios com alocação concentrada em poucos ativos possuem risco total elevado, dado que o impacto da porção não-sistemática do risco desses respectivos ativos na carteira não foi efetivamente minimizado por meio de diversificação. A partir da incorporação de mais ativos, o risco não-sistemático é passível de ser gradualmente minimizado, com o risco global do portfólio chegando a níveis próximos do risco sistemático, este comum a todos os ativos de risco.

Harry Markowitz, à época da publicação de seu revolucionário artigo "Portfolio Selection" no periódico Journal of Finance, em 1952, descobriu que a variância de um ativo por si só não era deveras relevante. Notou que a variância desse ativo em um portfólio é que traz maior riqueza para a análise de investimentos. A variância e o retorno esperado de um portfólio sem vendas a descoberto são mostrados nas Equações (1) e (2) (Markowitz, 1952; Elton, Gruber, Brown \& Goetzmann, 2012). 


$$
\begin{gathered}
\sigma_{P}^{2}=\sum_{j=1}^{N} X_{j}^{2} \sigma_{j}^{2}+\sum_{j=1}^{N} \sum_{\substack{k=1 \\
k \neq j}}^{N} \sigma_{j, k} X_{j} X_{k} \\
\overline{R_{P}}=\sum X_{i} \bar{R}_{i}
\end{gathered}
$$

Em que: $\overline{R_{P}}=$ Retorno esperado do portfólio; $\sigma_{\mathrm{P}}{ }^{2}=$ Variância do portfólio; $\overline{R_{l}}=$ Retorno esperado do ativo i; $\mathrm{X}_{\mathrm{i}}=$ Peso do ativo i; $\mathrm{X}_{\mathrm{j}}=$ Peso do ativo $\mathrm{j} ; \mathrm{X}_{\mathrm{k}}=$ Peso do ativo $\mathrm{k} ; \sigma_{\mathrm{j}}{ }^{2}=$ Variância do ativo $\mathrm{j} ; \sigma_{\mathrm{j}, \mathrm{k}}=$ Covariância dos ativos $\mathrm{j}$ e $\mathrm{k}$; $\mathrm{N}=$ Número de ativos

Estudos sobre diversificação já podiam ser encontrados no início do século XX, mas a novidade trazida por Markowitz $(1952,1959)$ adentrou na forma como o risco de um portfólio estava conectado com as covariâncias dos ativos individuais que o compõem. Desse modo, o autor consolidou o que era conhecimento convencional em investimentos e também gerou um processo pelo qual investidores poderiam escolher portfólios diversificados otimizados: a Análise Média-Variância (Damodaran, 2007).

\subsection{Análise média-variância}

A metodologia de seleção de portfólios proposta por Markowitz $(1952,1959)$ preocupa-se com duas dimensões: retorno esperado do portfólio e variância dos retornos do portfólio. Todas as combinações possíveis de ativos disponíveis para o investidor formam o conjunto possível de portfólios (feasible set). Entretanto, somente portfólios que oferecem o maior retorno esperado para determinado risco são portfólios eficientes. Consequentemente, para cada nível de risco há um portfólio eficiente. O conjunto desses portfólios eficientes é chamado de fronteira eficiente (Fabozzi \& Markowitz, 2011), equivalente ao exemplo exposto na Figura 2 a seguir:

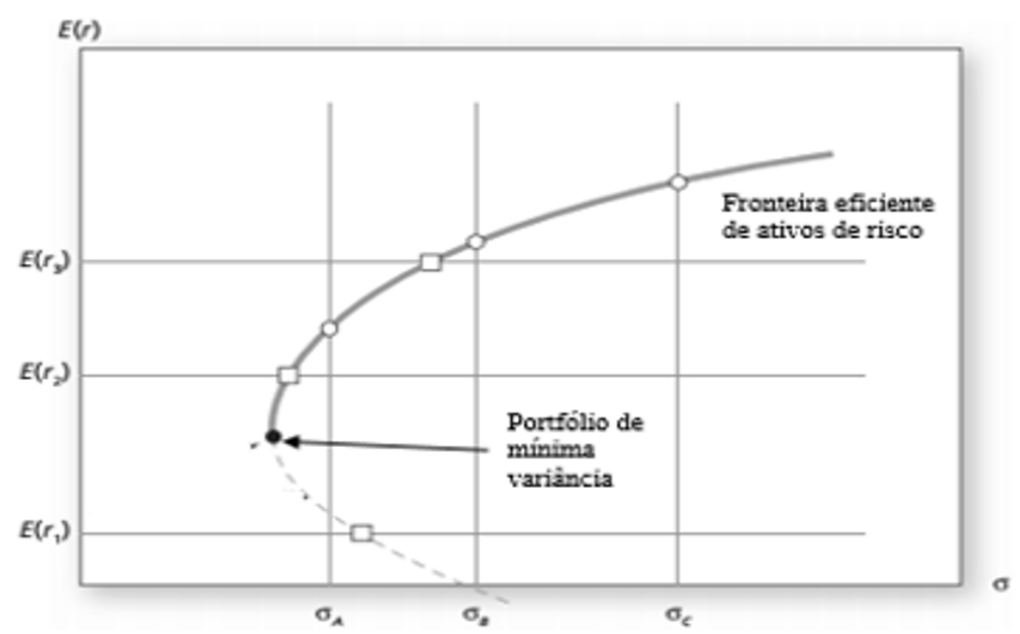

Figura 2. Fronteira eficiente sem ativo livre de risco

Fonte: Adaptado de Bodie, Kane e Marcus (2011)

Legenda: $\sigma_{\mathrm{A}}=$ Risco do Portfólio A; $\sigma_{\mathrm{B}}=$ Risco do Portfólio $\mathrm{B} ; \sigma_{\mathrm{C}}=$ Risco do Portfólio C; $\mathrm{E}\left(\mathrm{r}_{1}\right)=$ Retorno Esperado do Portfólio 1; $\mathrm{E}\left(\mathrm{r}_{2}\right)=$ Retorno Esperado do Portfólio 2; $\mathrm{E}\left(\mathrm{r}_{3}\right)=$ Retorno Esperado do Portfólio 3

Caso um ativo livre de risco seja introduzido como opção de investimento, a fronteira eficiente passará a ser uma reta que tangencia a fronteira eficiente de ativos de risco. A reta chama-se Reta do Mercado de Capitais e representa as opções de composição do ativo livre de risco com um portfólio que represente o mercado de capitais como um todo (Fabozzi \& Markowitz, 2011). A fronteira eficiente com ativo livre de risco, por sua vez, é ilustrada na Figura 3. 


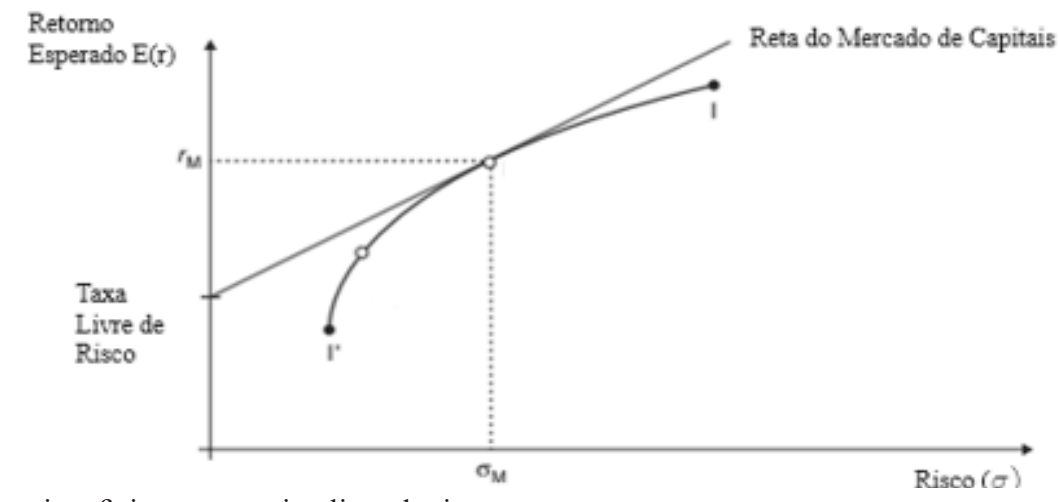

Figura 3. Fronteira eficiente com ativo livre de risco

Fonte: Adaptado de Vernimmen et al. (2014)

Legenda: $\sigma_{\mathrm{M}}=$ Risco do Portfólio de Mercado; $\mathrm{r}_{\mathrm{M}}=$ Retorno Esperado do Portfólio de Mercado

A metodologia Média-Variância possui duas premissas básicas: a normalidade das distribuições dos retornos dos ativos e que as funções de utilidade de todos os investidores são quadráticas. Damoradan (2007) refuta a premissa sobre retornos, afirmando que a maioria dos investimentos não possuem retornos normalmente distribuídos e simétricos. Elton e Gruber (1997) corroboram na configuração deste método ao mostrar como a Análise Média-Variância é sensível, mostrando dependência da forma como os inputs (retorno esperado e variância dos ativos) da análise são calculados. Ainda afirmam que o tamanho das séries históricas a serem utilizadas é um tópico significativo e que poucas pesquisas foram realizadas sobre o assunto.

\section{3 Value-at-Risk e Conditional Value-at-Risk}

Value-at-Risk (VaR) e sua adaptação Conditional Value-at-Risk (CVaR) são medidas de risco downside. Estas vertentes preocupam-se com a magnitude das perdas possíveis pelo investidor (Dempster, 2002). A terminologia risco downside foi primeiramente estudada por Roy (1952) na sequência das publicações de Harry Markowitz sobre Média-Variância. Roy (1952) criticou as funções de utilidade e a obscuridade de prever a utilidade dos investidores, propondo que investidores preferem investimentos com as menores chances de perdas extremas (Nawrocki, 1999, como citado em Araújo, 2011). Jorion (2006) define VaR como uma medida da maior potencial perda em valor de um ativo de risco ou portfólio em um dado período de tempo para um dado intervalo de confiança. A formulação matemática não-paramétrica do VaR é mostrada na Equação (3).

$$
1-c=\int_{-\infty}^{V a R} f(w) d w=P(w \leq \operatorname{VaR})
$$

Em que: $c$ = Intervalo de confiança; $\mathrm{f}(\mathrm{w})=$ Função densidade de probabilidade do valor futuro do portfólio

As possibilidades de especificação de $\mathrm{f}(\mathrm{w})$ são inúmeras, e cada uma possui suas premissas, vantagens e desvantagens (Damodaran, 2007). Artzner, Delbaen, Eber e Heath (1997,1999), adicionalmente, propõem um conjunto de axiomas que constituem propriedades para que uma medida de risco seja considerada coerente. Os axiomas são: monotocidade, subaditividade, homogeneidade positiva e invariância à translação (Acerbi \& Tasche, 2002). Assim, apesar do tremendo sucesso do VaR, críticas surgiram no final da década de 1990, sendo duas as principais: o VaR mede apenas os percentis da distribuição de perdas, ignorando perdas além do nível de VaR (chamado de tail risk); e, não é uma medida coerente de risco, pois não é subaditivo (Artzner et al., 1997, 1999; Yamai \& Yoshiba, 2005).

Acerbi (2002) aponta que subaditividade é essencial para uma medida de risco, pois esse axioma captura a 
essência de como um portfólio feito de sub-portfólios terá seu risco mensurado. Com a violação da subaditividade, o risco de um portfólio poderá ser maior do que a soma de suas partes, trazendo resultados não-ótimos para a gestão de risco.

Para contornar o problema de tail risk e de subaditividade, Artzner et al. $(1997,1999)$ propõem uma nova medida de risco: o CVaR (também chamado de expected shortfall, tail Value-at-Risk ou mean shortfall). Satisfazendo todos os axiomas de uma medida coerente de risco, o CVaR pode ser definido como a média ponderada das expectativas de perdas quando essas são maiores que o VaR (Moreira, 2006). A formulação do CVaR é mostrada na Equação (4) e a comparação das propriedades do VaR e CVaR é mostrada no Tabela 1.

$$
C \operatorname{VaR}^{\alpha}=E\left(P \mid P>\operatorname{VaR}^{\alpha}\right)
$$

Em que: $\alpha=$ Intervalo de confiança; $\mathrm{P}=$ Lucro/perda do portfólio; $\mathrm{VaR}^{\alpha}=\mathrm{VaR}$ com intervalo de confiança

Tabela 1. Comparação entre VaR e CVaR

\begin{tabular}{ccc}
\hline Propriedade & VaR & CVar \\
\hline Monotocidade & Sim & Sim \\
Subaditividade & Não & Sim \\
Homogeneidade positiva & Sim & Sim \\
Invariância à translação & Sim & Sim \\
\hline
\end{tabular}

Fonte: Elaborado pelos autores

Subsequentemente, Rockafellar e Uryasev $(2000,2002)$ realizaram avanços em otimização envolvendo o $\mathrm{CVaR}$, chegando à conclusão que o problema pode ser resolvido por programação estocástica e linear. A abordagem consiste em cálculos simultâneos do CVaR e VaR, observando que a otimização do CVaR também implica na otimização do VaR (pois o CVaR é sempre maior ou igual ao VaR). Recentes estudos desenvolvimentos sobre o CVaR apontam que a nova métrica é mais sensível que o VaR quanto a erros na estimação dos inputs. Assim, o sucesso do CVaR está altamente ligado à precisão da modelagem da distribuição do risco acima do VaR (tail risk) (Uryasev, 2010).

\subsection{Fundos de Investimento Imobiliário}

Os FIIs foram criados pela Lei $n^{0}$ 8.668/93, com as Instruções da Comissão de Valores Mobiliários (CVM) 205/94 e 206/94, contendo as diretrizes e normas para funcionamento e distribuição. A referência na época eram os REITs existentes nos Estados Unidos, apesar dos FIIs não terem absorvido todas as suas características (Decreto-Lei n. 8.668, de 25 de junho de 1993, \& ANBIMA, 2014). A última revisão da regulamentação define os FIIs como: " é uma comunhão de recursos captados por meio do sistema de distribuição de valores mobiliários e destinados à aplicação em empreendimentos imobiliários. O fundo será constituído sob a forma de condomínio fechado e poderá ter prazo de duração indeterminado" (Instrução CVM n. 472, 2008, p. 1).

Os lucros provenientes dos FIIs distribuídos para investidores Pessoa Física também são isentos de Imposto de Renda, desde que 95\% dos lucros sejam distribuídos aos cotistas; com um mínimo de 50 cotistas, sendo que nenhum cotista Pessoa Física possua mais de 10\% das cotas do fundo; e nenhuma parte relacionada (construtoras, incorporadoras) tenha mais de 25\% das cotas do fundo (Instrução CVM n. 472, 2008).

Em termos de suas configurações, os FIIs são classificados como fundos de investimento fechados. Desse modo, para que um investidor venda sua cota (fração ideal do FII), é necessário o acesso aos mercados secundários. Por norma, todos os FIIs devem possuir negociação em bolsa de valores ou mercado de balcão organizado. Tais fundos podem ainda ser classificados de acordo com os ativos que compõem seu portfólio: Fundos de Renda, Fundos de Desenvolvimento e Fundos de Ativos Financeiros.

O Índice de Fundos de Investimento Imobliário (IFIX), por sua vez, é um índice setorial de mercado 
criado pela BM\&FBOVESPA com o objetivo de ser o indicador de desempenho médio dos FIIs listados nos mercados de bolsa de valores e de balcão organizado. Desse modo, o IFIX reflete a dinâmica do mercado como um todo, sendo o primeiro benchmark do setor (BM\&FBOVESPA, 2015; UQBAR, 2015). Ele foi criado em setembro de 2012, e a sua série histórica pode ser acompanhada retroativamente desde dezembro de 2010, quando o índice foi fixado em 1.000 pontos por sua criadora.

Sendo um índice de retorno total, o IFIX não reflete apenas a variação no preço das cotas de FIIs, mas também dividendos, juros sobre o capital próprio, direitos de subscrição e bonificações. Assim, o valor total da carteira teórica do IFIX é composto pelos preços "ex-teóricos" dos ativos que a compõem (BM\&FBOVESPA, 2015).

\subsection{Indicadores de Real Estate Investment Trusts (REIT)}

REITs norte-americanos foram a referência para a criação dos FIIs. Sua concepção data do final do século XIX, mas foi em 1960 que o Congresso dos Estados Unidos aprovou as legislações necessárias para sua regulamentação, procurando incentivar e pulverizar novos investimentos no setor imobiliário (Brueggerman \& Fisher, 2008; Calado, Giotto, \& Securato, 2001).

Podem ser classificados em três grandes tipos: Equity REITs (investimento de participação em imóves), Mortgage REITs (investimento por meio de dívida) e Hybrid REITs (mescla entre Equity e Mortgage). Ao final de 2014 havia 203 REITs com capitalização de mercado combinada de 830 bilhões de dólares (FTSE, 2015; Block, 2006). Apesar das semelhanças, FIIs e REITs possuem algumas diferenças. REITs são incorporados como empresas, devem possuir pelo menos 100 acionistas, 5 maiores acionistas não podem possuir mais de $50 \%$ de participação no REIT e 90\% dos lucros devem ser distribuídos (Cosentino \& Alencar, 2011).

Block (2006) e Brueggeman e Fisher (2008) citam dois importantes indicadores para avaliação de REITs: Preço/Funds from Operations (P/FFO) e Net Asset Value Premium (P/NAV). FFO, uma medida não-contábil, é utilizado como métrica do fluxo de caixa gerado pelo REIT disponível para distribuição aos acionistas. FFO é preferível (ao invés do Lucro Líquido) devido ao impacto da depreciação e receitas não-recorrentes no fluxo de caixa. O múltiplo valor de mercado do REIT dividido pelo FFO (ou preço da ação dividido pelo FFO por ação) proporciona uma análise comparativa com REITs similares. A fórmula para o FFO e o múltiplo P/FFO são encontradas nas Equações (5) e (6), respectivamente.

$\mathrm{P} / F F O=$ Valor de Mercado do REIT

FFO

Net Asset Value (NAV) é o valor de mercado estimado de todos os ativos do REIT depois de subtraídas todas as obrigações e passivos. Deve ser estimado por analistas e investidores já que os REITs não possuem avaliações periódicas dos preços de mercado dos imóveis que compõem seus portfólios (Block, 2006). O P/NAV procura avaliar se os ativos do REIT estão sobre ou subavaliados. As fórmulas para o NAV e NAV-P são mostradas nas Equações (7) e (8), respectivamente.

NAV=Valor de mercado das propriedades do REIT - Dívida

$\mathrm{P} / \mathrm{NAV}=$ Valor de Mercado do REIT 


\section{METODOLOGIA}

\subsection{Determinação dos modelos}

Para conduzir a comparação entre os dois diferentes tipos de modelos, risco-variância e risco downside, foram escolhidos o modelo de Markowitz (1952) para risco-variância e a otimização do CVaR proposta por Rockafellar e Uryasev $(2000,2002)$ para risco downside. O CVaR foi preferível ao VaR por possuir propriedades matemáticas mais favoráveis à otimização e pela importância do tail risk, conforme apresentado na Tabela 1. As funções objetivas e restrições dos modelos são apresentadas na Tabela 2.

Tabela 2. Modelos utilizados para comparação

\begin{tabular}{cc}
\hline Markowitz (1952) & Rockafellar e Uryasev $(2000,2002)$ \\
\hline Minimizar: & Minimizar: \\
$\sum_{i=1}^{n} \sum_{j=1}^{n} w_{i} w_{j} \sigma_{i j}$ & $\alpha+\frac{1}{q(1-\beta)} \sum_{k=1}^{q}\left[-w^{T} y_{k}-\alpha\right]^{+}$ \\
Sujeito a: & Sujeito a: \\
$\sum_{i=1}^{n} w_{i}=1$ & $\sum_{i=1}^{n} w_{i}=1$ \\
$\sum_{i=1}^{n} w_{i} R_{i}=R_{w}$ & $\sum_{i=1}^{n} w_{i} R_{i}=R_{w}$ \\
$0 \leq w_{i} \leq 1$ & $0 \leq w_{i} \leq 1$ \\
\hline
\end{tabular}

Fonte: Markowitz (1952) e Rockafellar e Uryasev (2000, 2002)

Em que:

$\mathrm{w}_{\mathrm{i}}=$ Ponderação do ativo "i” no portfólio; $\sigma_{i j}=$ Covariância dos ativos " $\mathrm{i}$ ” e " $\mathrm{j}$ ”; $\mathrm{R}_{\mathrm{i}}=$ Retorno esperado do ativo " $\mathrm{i}$ ”; $\mathrm{R}_{\mathrm{iw}}=$ Retorno esperado do portfólio; $\beta=$ Nível de probabilidade das perdas máximas; $\alpha=$ Value-at-Risk máximo; $\mathrm{y}_{\mathrm{k}}=$ Vetor de perdas do cenário "k"; $q=$ Número de cenários

\subsection{Amostra e período analisado}

Após a definição dos modelos a serem implementados, o próximo passo consiste na definição dos ativos que serão elegíveis para a composição do Fundo de FIIs e do período de estudo. Procurou-se como princípios norteadores de elegibilidade a liquidez oferecida pelo FII, a natureza de seus investimentos e a disponibilidade de dados. Em 18 de julho de 2015, o IFIX, índice que mede a performance dos FIIs mais líquidos e significativos presentes no âmbito da BM\&FBOVESPA, possuía em sua carteira teórica quarenta e seis FIIs que são negociados em bolsa de valores. Dentre estes, três FIIs são fundos de FIIs que serão desconsiderados da amostra.

Analisando a disponibilidade de dados (condição de pelo menos 90\% de cotações presentes no período), estudos anteriores e revisão de literatura, decidiu-se por utilizar o período de estudo de dois anos, de julho de 2013 a julho de 2015. As cotações de fechamento ajustadas para dividendos e proventos foram acessadas por meio do banco de dados do software Economatica. Dos quarenta e três FIIs selecionados, apenas trinta possuem mais de $90 \%$ de cotações diárias no período, os quais compõem 66,2\% do IFIX. Finalmente, os FIIs elegíveis e a distribuição por classe de ativo são mostrados na Tabela 3. 
Tabela 3. FIIs elegíveis

\begin{tabular}{|c|c|c|}
\hline Código & Nome & Classe \\
\hline AEFI11 & AESAPAR & Escolar \\
\hline AGCX11 & Agências Caixa & Agências Bancárias \\
\hline BBPO11 & BB Progressivo II & Agências Bancárias \\
\hline BBRC11 & BB Renda Corporativa & Agências Bancárias \\
\hline BBVJ11 & $\begin{array}{c}\text { BB Votorantim JHSF } \\
\text { Cidade Jardim Continental } \\
\text { Tower }\end{array}$ & Escritórios \\
\hline BRCR11 & $\begin{array}{c}\text { BTG Pactual Corporate } \\
\text { Office }\end{array}$ & Escritórios \\
\hline FFCI11 & $\begin{array}{l}\text { Rio Bravo Renda } \\
\text { Corporativa }\end{array}$ & Escritórios \\
\hline FIGS11 & $\begin{array}{c}\text { General Shopping Ativo e } \\
\text { Renda }\end{array}$ & Shopping Centers \\
\hline FLMA11 & $\begin{array}{c}\text { Continental Square Faria } \\
\text { Lima }\end{array}$ & Escritórios \\
\hline HGBS11 & CSHG Brasil Shopping & Shopping Centers \\
\hline HGLG11 & CSHG Logística & Logística \\
\hline HGRE11 & CSHG Real Estate & Escritórios \\
\hline KNCR11 & $\begin{array}{l}\text { Kinea Rendimentos } \\
\text { Imobiliários }\end{array}$ & Ativos Financeiros \\
\hline KNRI11 & Kinea Renda Imobiliária & Logística \\
\hline MXRF11 & Maxi Renda & Ativos Financeiros \\
\hline PQDP11 & $\begin{array}{l}\text { Parque Dom Pedro } \\
\text { Shopping Center }\end{array}$ & Shopping Centers \\
\hline PRSV11 & Presidente Vargas & Escritórios \\
\hline RBGS11 & $\begin{array}{l}\text { RB Capital General } \\
\text { Shopping Sulacap }\end{array}$ & Shopping Centers \\
\hline RBRD11 & RB Capital Renda II & Logística \\
\hline RNGO11 & Rio Negro & Escritórios \\
\hline SAAG11 & Santander Agências & Agências Bancárias \\
\hline SDIL11 & SDI Logística RIO & Logística \\
\hline SPTW11 & SP Downtown & Escritórios \\
\hline TBOF11 & TB Office & Escritórios \\
\hline TRXL11 & $\begin{array}{l}\text { TRX Realty Logística } \\
\text { Renda I }\end{array}$ & Logística \\
\hline VLOL11 & Vila Olímpia Corporate & Escritórios \\
\hline VRTA11 & Fator Verita & Ativos Financeiros \\
\hline XPCM11 & XP Corporate Macaé & Escritórios \\
\hline XPGA11 & XP Gaia Lote I & Ativos Financeiros \\
\hline XTED11 & TRX Edifícios Corporativos & Escritórios \\
\hline
\end{tabular}

Fonte: Elaborado pelos autores

\subsection{Tratamento dos dados}

Definidos os FIIs elegíveis, intervalo de retorno e período do estudo, o próximo passo compreende 
exportar as cotações diárias de fechamento ajustadas do software Economatica para uma planilha eletrônica usando o software Microsoft Excel. As cotações exportadas foram de 19 de julho de 2013 a 20 de julho de 2015, consistindo em 496 dias de negociações. A partir das cotações, os retornos diários foram obtidos pela Equação (2). Em eventuais dias, alguns ativos não foram negociados e não possuem possibilidade de cálculo de seus retornos. Para contornar esse obstáculo, nas datas em que não há cotações, foram mantidas as cotações do dia imediatamente anterior.

Para a aplicação dos indicadores de REITs aos FIIs, lançou-se mão das últimas demonstrações financeiras auditadas de cada FII cadastradas no sistema BM\&FBOVESPA. Por peculiaridades legais e diferenças nas estruturas societárias, algumas adaptações nos métodos de cálculo foram realizados. Como os FIIs não são autorizados a depreciarem seus ativos, para o cálculo do FFO foram descontadas do lucro líquido todas as receitas e despesas não recorrentes, quais sejam, as relacionadas a vendas de ativos (salvo quando objetivo de investimento do FII) e ajustes do valor dos imóveis.

O NAV de cada FII foi estimado pelo seu patrimônio líquido. Os métodos de cálculo adotados para os indicadores aplicados aos FIIs são apresentados nas Equações (9) e (10).

$$
\begin{gathered}
\text { FFO = Lucro Líquido-Ganho (Perda) com Vendas de Ativos - Ganho (Perda) } \\
\text { em Ajuste do Valor dos Imóveis } \\
\text { NAV = Ativo - Passivo }
\end{gathered}
$$

\subsection{Restrições}

Restrições na composição dos portfólios definem os limites das ponderações relativas de cada ativo permitidas para otimização. No estudo foram realizadas quatro tipos de restrições, todas sem a permissão de vendas a descoberto.

\subsubsection{Todos os ativos}

Dois portfólios estão presentes nesta classificação: Fundo "Global” e Fundo "Limites por Emissor". O primeiro permite que os ativos tenham qualquer peso entre zero e um, enquanto que o posterior segue os limites impostos pela CVM quanto à concentração do patrimônio líquido do fundo quando o emissor for outro fundo de investimento (Instrução CVM nº. 409, 2004).

\subsubsection{Restrições relacionadas ao beta}

Nesta categoria, o Beta em relação ao IFIX age como divisor entre FIIs com diferentes níveis de risco. A separação foi realizada por meio da mediana dos coeficientes Beta de todos os FIIs elegíveis. Dois fundos compõem esse tipo de restrição: Fundo "Beta < Mediana" e Fundo "Beta > Mediana".

\subsubsection{Restrições relacionadas aos indicadores de REITs}

Com os indicadores $\mathrm{P} / \mathrm{FFO}$ e $\mathrm{P} / \mathrm{NAV}$, os FIIs foram divididos em grupos de acordo com a mediana de cada indicador. As capitalizações de mercado utilizada para o cálculo do P/FFO e P/NAV foram as últimas contidas na série histórica utilizada para a otimização, as capitalizações do dia 20 de julho de 2015 . A Tabela 4 contém os

valores dos indicadores para cada FII. 
Tabela 4. P/FFO e P/NAV

\begin{tabular}{lrrlrr}
\hline FII & P/FFO & P/NAV & FII & P/FFO & P/NAV \\
\hline BRCR11 & 9,8 & 0,7 & VLOL11 & 9,3 & 0,6 \\
AEFI11 & 8,6 & 0,7 & PRSV11 & 5,2 & 0,5 \\
AGCX11 & 10,9 & 0,9 & RBGS11 & 6,0 & 0,5 \\
BBRC11 & 34,1 & 0,9 & RBRD11 & 9,3 & 1,0 \\
BBPO11 & 10,2 & 1,1 & FFCI11 & 10,2 & 0,8 \\
BBVJ11 & 11,4 & 0,5 & RNGO11 & 8,3 & 0,9 \\
HGLG11 & 16,1 & 1,0 & FLMA11 & 11,0 & 0,6 \\
HGBS11 & 10,8 & 0,8 & SAAG11 & 10,3 & 0,9 \\
PQDP11 & 6,1 & 0,6 & SDIL11 & 8,6 & 0,8 \\
VRTA11 & 11,0 & 1,0 & SPTW11 & 66,0 & 0,7 \\
FIGS11 & 7,6 & 0,7 & TBOF11 & 8,6 & 0,6 \\
HGRE11 & 13,6 & 0,9 & TRXL11 & 7,7 & 0,7 \\
KNRI11 & 11,1 & 0,8 & XTED11 & 5,8 & 0,6 \\
KNCR11 & 17,5 & 1,6 & XPGA11 & 3,6 & 0,9 \\
MXRF11 & 10,0 & 0,9 & XPCM11 & 11,9 & 0,8 \\
Mediana & $\mathbf{1 0 , 1}$ & $\mathbf{0 , 8}$ & & & \\
\hline Fon: Elab & & & & &
\end{tabular}

Fonte: Elaborado pelos autores

\subsubsection{Restrições relacionadas à classe de ativo}

A restrição por classe de ativo é composta por um fundo isonômico entre os diferentes tipos de empreendimentos de base imobiliária existentes. Para cada classe foi definido um FII representante, sendo que esses ativos foram selecionados de acordo sua relação risco e retorno. O portfólio foi denominado Fundo "Iso".

\subsection{Otimização dos modelos}

Com os dados organizados adequadamente e as restrições pré-determinadas, o próximo passo consistiu em otimizar os modelos. O retorno esperado de cada FII foi calculado pela média histórica dos retornos. Ambas as otimizações Média-CVaR (intervalo de confiança de 95\%) e Média-Variância foram realizadas pelo software MathWorks MATLAB. Para cada uma das otimizações, são utilizados dois tipos diferentes de solver: programação estocástica para a Média-CVaR e programação quadrática para a Média-Variância (MathWorks, 2015). Para realizar a comparação entre as diferentes métricas de risco, os portfólios otimizados por Média-CVaR tiveram seus desvios-padrão calculados. A partir dos dados obtidos para cada portfólio de cada restrição (pesos, retornos esperados e desvios-padrão), as fronteiras eficientes foram plotadas por meio do software Microsoft Excel.

\section{RESULTADOS E DISCUSSÃO}

Neste capítulo são apresentadas as fronteiras eficientes para as diversas restrições calculadas a partir das otimizações Média-CVaR e Média-Variância com cotações históricas dos 30 FIIs elegíveis de julho de 2013 a julho de 2015. 


\subsection{Todos os ativos}

A Figura 4 apresenta as fronteiras eficientes para o Fundo “Global”, sem restrições. Também são plotados alguns FIIs selecionados para referência. A seleção procurou identificar os FIIs com as melhores relações risco e retorno, razão formulada na Equação (5). Também é destacado o Portfólio de Mínima Variância (PMV).

$$
R R_{i}=\frac{\bar{R}_{i}}{\sigma_{i}}
$$

Em que:

$\mathrm{RR}_{\mathrm{i}}=$ Relação Risco-Retorno; $\bar{R}_{\boldsymbol{\iota}}=$ Retorno esperado do ativo “i”; $\sigma_{\mathrm{i}}=$ Desvio-padrão do ativo "i”

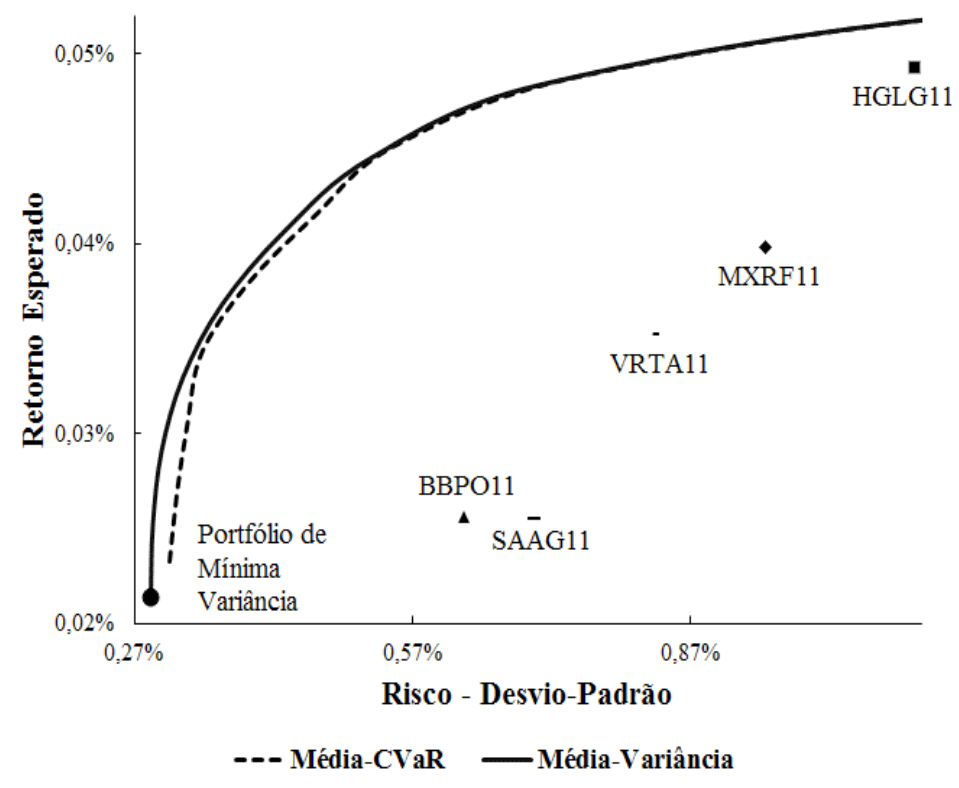

Figura 4. Fronteiras eficientes do Fundo "Global"

Fonte: Elaborado pelos autores

Todos os portfólios otimizados são eficientes, ou seja, possuem maior retorno para um mesmo risco, frente à carteira teórica do IFIX. A diferença marcante entre a otimização Média-CVaR para a Média-Variância é a discrepância das fronteiras em níveis de baixo risco. Isso ocorre porque a otimização Média-CVaR procura remodelar as perdas extremas, enquanto que a Média-Variância as trata como uma distribuição normal.

Quando comparados portfólios de mesmo retorno esperado, a otimização Média-Variância fornece portfólios com até $10 \%$ menos variância que portfólios Média-CVaR. Essa diferença tende a desaparecer conforme o nível de risco aumenta, sugerindo que a otimização Média-Variância subestima as chances de grandes perdas (tail risk). Analogamente, as fronteiras eficientes do Fundo "Limites por Emissor" são mostradas na Figura 5. 


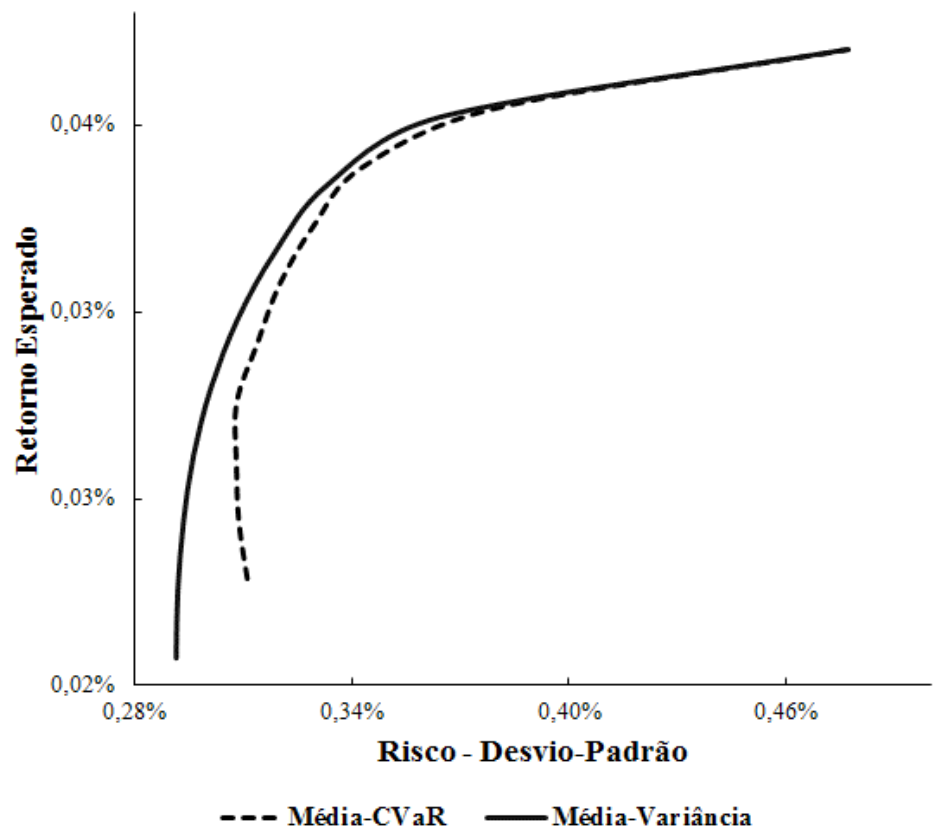

Figura 5. Fronteiras eficientes do Fundo "Limites por Emissor"

Fonte: Elaborado pelos autores

Do mesmo modo que o Fundo "Global”, as diferenças aparentes entre as métricas de risco são mais notáveis nos níveis mais baixos de risco. Com a imposição dos limites regulatórios por emissor, os portfólios possuem menor capacidade de auferir altos retornos. O retorno anualizado máximo do Fundo "Limites por Emissor" é de 10,89\% frente aos 16,35\% possíveis com o Fundo "Global”. Também é possível observar que alguns portfólios obtidos pela Média-CVaR não são eficientes do ponto de vista do risco variância.

\subsection{Restrições relacionadas ao beta}

Na Figura 6 são apresentadas quatro fronteiras eficientes: Média-CVaR e Média-Variância dos Fundos "Beta $<$ Mediana" e "Beta $>$ Mediana".

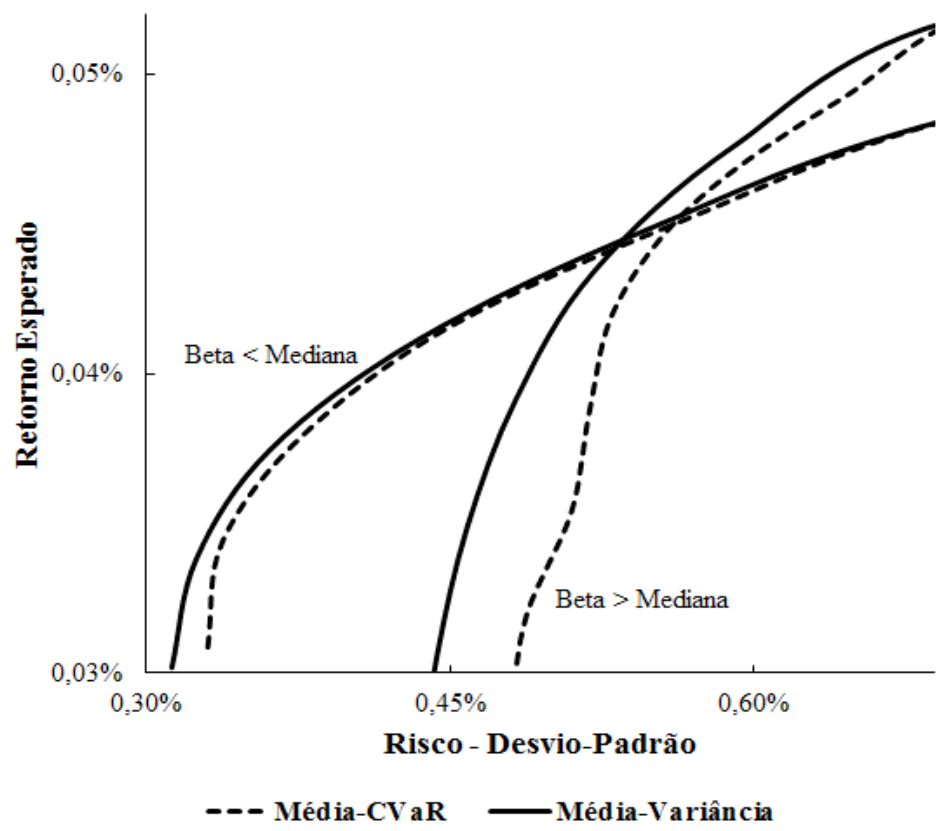

Figura 6.Fronteiras eficientes dos Fundos "Beta $<$ Mediana" e "Beta $>$ Mediana" Fonte: Elaborado pelos autores. 
Os pontos de interseção representam quando uma fronteira passa a ser dominante sobre a outra. Assim, um investidor que inicialmente procurava investir na fronteira do Fundo "Beta $<$ Mediana", irá preferir mudar para a fronteira do Fundo "Beta > Mediana" a partir do nível de risco em que ocorrerá a interseção da primeira com a segunda fronteira (referente às duas métricas em análise). As otimizações apresentam maiores diferenças entre si quando os ativos possuem Betas maiores, chegando a 15\% de diferença relativa para um mesmo retorno esperado.

\section{3 Restrições relacionadas aos indicadores de REITs}

As fronteiras eficientes para os Fundos "Crescimento" e "Desconto" foram plotadas no mesmo gráfico para comparação. Cada uma das restrições possui 15 FIIs. As fronteiras são mostradas na Figura 7.

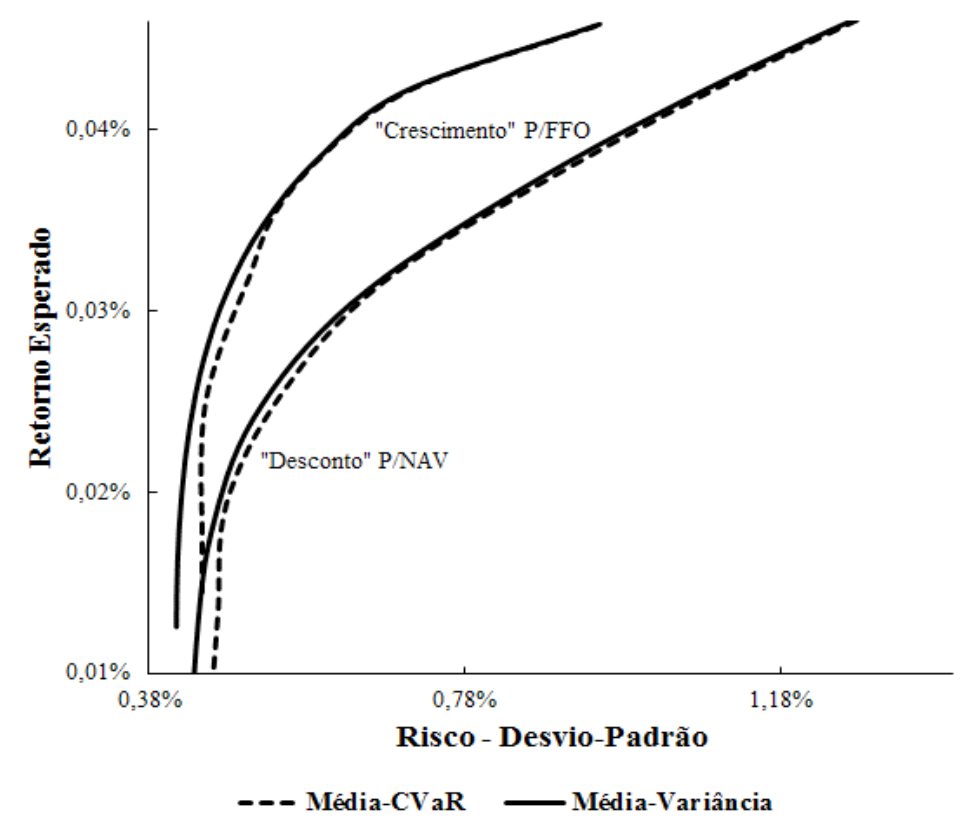

Figura 7. Fronteiras eficientes dos Fundos "Crescimento" e "Desconto"

Fonte: Elaborado pelos autores

Os portfólios compostos pelos ativos do Fundo "Crescimento" foram sempre eficientes em relação aos do Fundo "Desconto", mesmo que retornos esperados maiores possam ser atingidos pelo Fundo "Desconto". Assim, evidencia-se que o indicador P/FFO aparenta ser mais adequado para a avaliação de FIIs, ao invés do indicador P/ NAV. Entretanto, a diferença relativa entre as duas métricas foi levemente acentuada para os portfólios do Fundo "Crescimento", chegando a 8\% para um mesmo retorno esperado no nível mais baixo de risco.

\subsection{Restrições relacionadas à classe de ativo}

O Fundo "Iso" foi composto por um FII de cada classe, quais sejam: Logística, Agências Bancárias, Ativos Financeiros, Escritórios, Shopping Centers e Escolar. Os ativos foram selecionados pelas melhores relações risco e retorno, conforme Equação (5). A fronteira eficiente, os pontos que representam os FIIs elegíveis e o índice IFIX são mostrados na Figura 8. 


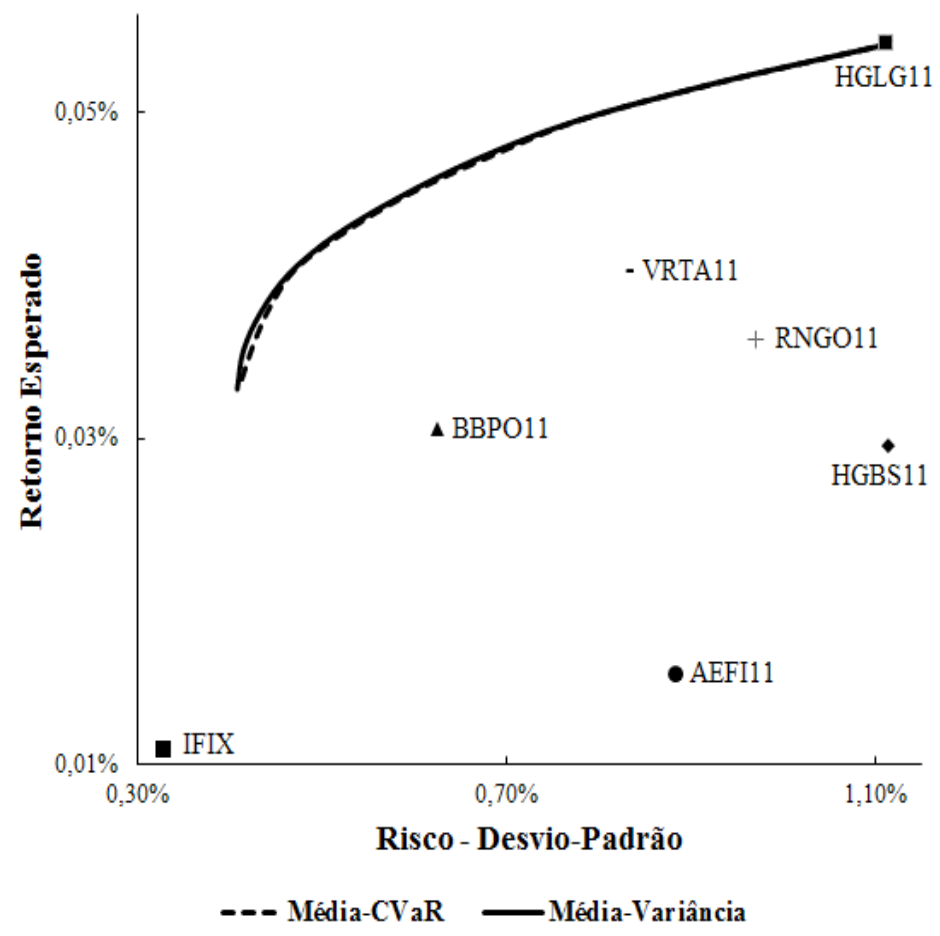

Figura 8. Fronteiras eficientes do Fundo "Iso"

Fonte: Elaborado pelos autores

Apenas com os FIIs do Fundo "Iso" não foi possível obter um portfólio com o nível de risco do IFIX. O PMV desta restrição possui desvio-padrão diário de $0,41 \%$, enquanto que o IFIX possui $0,33 \%$. Entretanto, os portfólios dessa fronteira eficiente foram capazes de produzir retornos maiores para uma mesma taxa de risco, comparados às carteiras dos Fundos "Limites por Emissor", "Crescimento" e "Desconto". Cabe salientar que essa restrição foi a que menos apresentou diferenças entre as otimizações, com diferença relativa máxima de 3,12\% de risco para um mesmo retorno esperado.

\section{CONSIDERAÇÕES FINAIS}

Sendo o objetivo primordial deste trabalho a análise comparativa de diferentes métricas de risco na otimização de um Fundo de FIIs, lançou-se mão de modelos pertencentes a MPT e PMPT. A metodologia empregada envolveu o levantamento dos principais modelos utilizados, como métricas de risco de mercado, seleção da amostra e período de análise e restrições adicionais aos modelos. Foram analisados 30 FIIs listados na bolsa de valores da BM\&FBOVESPA entre julho de 2013 a julho de 2015, compreendendo 496 dias de negociações.

Observou-se que nos níveis mais baixos de risco as métricas de risco CVaR e Variância apresentaram diferentes resultados. Por ser uma métrica de risco downside, o CVaR procura minimizar perdas extremas (cauda esquerda, tail risk). Desta forma, mesmo com retorno esperado menor na comparação à otimização Média-Variância (quando comparando os portfólios pelos seus respectivos desvios-padrão e retorno esperado), ele é mais indicado a investidores interessados nos menores riscos de risco possíveis, visto que o tail risk está minimizado - embora com menor potencial de retorno esperado. Essas perdas foram subestimadas pela variância, visto que essa assume a distribuição normal dos retornos. Assim, para investidores mais interessados em investir nos mais baixos níveis de risco, sugere-se a composição de um Fundo de FIIs a partir dos portfólios gerados pela otimização Média-CVaR. A partir de certo nível de risco, as duas métricas convergem para os mesmos resultados.

Para futuros estudos, recomenda-se a análise do impacto de outros tipos de títulos com lastro imobiliário no desempenho dos Fundos de FIIs. Sugere-se também, quando disponível, a análise com um maior período de estudo e maior amostra de FIIs, de modo a caracterizar de forma mais profunda o comportamento das distribuições de retornos dos FIIs. 


\section{REFERÊNCIAS}

Acerbi, C. (2002). Spectral Measures of Risk: a Coherent Representation of Subjective Risk Aversion. Journal of Banking \& Finance, 26(7), 1505-1518.

Acerbi, C. \& Tasche, D. (2002). On the coherence of expected shortfall. Journal of Banking \& Finance, 25(7), 1487-1503.

Associação das Entidades dos Mercados Financeiro e de Capitais. (2014). Fundos de Investimento Imobiliário. Rio de Janeiro: Estudos Especiais: Produtos de Captação.

Araújo, A. C. (2011). Comparação entre métricas de risco para otimizar carteiras de investimentos em ações (Dissertação de mestrado). Faculdade de Economia, Administração e Contabilidade da Universidade de São Paulo, São Paulo, SP, Brasil.

Artzner, P., Delbaen, F., Eber, J. M., \& Health, D. (1997). Thinking coherently. Risk, 10, 68-71.

Artzner, P.; Delbaen, F.; Eber, J. \& Heath, D. (1999). Coeherent measures of risk. Mathematical Finance. 9(3), 203-228.

Berk, J.; Demarzo, P. \& Harford, J. (2012). Fundamentals of Corporate Finance. Boston: Prentice Hall.

Block, R. L. (2006) Investing in REITs. New York: Bloomberg Press.

BM\&FBOVESPA. (2015) Metodologia do Índice de Fundos de Investimento Imobiliário (IFIX). São Paulo: Autor.

Bodie, Z., Kane A., \& Marcus, A. J. (2011). Investments. New York: McGraw-Hill/Irwin.

Brueggeman, W. B., \& Fisher, J. D. (2008). Real Estate Finance and Investments. New York: McGraw-Hill.

Calado, L. R., Giotto, R. M., \& Securato, J. R. (2001). Um estudo atual sobre fundos de investimento imobiliário. Anais do V SEMEAD. São Paulo, SP, Brasil.

Cosentino, R. M. S. \& Alencar, C. T. (2011). Fundos de Investimento Imobiliário: Análise do desempenho e comparação com US-REITs, UK-REITs, G-REITs e SIIC. Anais da Conferência Internacional da LARES, São Paulo, SP, Brasil, 11.

Instrução CVM n. 409. (2004). Dispõe sobre a constituição, a administração, o funcionamento, a oferta pública de distribuição de cotas e a divulgação de informações dos Fundos de Investimento Imobiliário - FII. Revoga as Instruções CVM n. 205, de 14 de janeiro de 1994, n. 389, de 3 de junho de 2003, n. 418, de 19 de abril de 2005 e n. 455, de 13 de junho de 2007. Acrescenta o Anexo III-B à Instrução CVM n. 400, de 29 de dezembro de 2003. Recuperado de http://www.cvm.gov.br/legislacao/i nst/inst409.html

Instrução CVM n. 472. (2008). Dispõe sobre a constituição, a administração, o funcionamento, a oferta pública de distribuição de cotas e a divulgação de informações dos Fundos de Investimento Imobiliário - FII. Revoga as instruções CVN n. 205, de 14 de janeiro de 1994, n. 389, de 3 de junho de 2003, n. 418, de 19 de abril de 2005 e n. 455, de 13 de junho de 2007. Acrescenta o Anexo III-B à Instrução CVM n. 400, de 29 de dezembro de 2003. Recuperado de http://www.cvm.gov.br/legislacao/i nst/inst472.html

Damodaran, A. (2007). Strategic Risk Taking: A Framework for Risk Management. London: FT Press.

Decreto-Lei n. 8.668, de 25 de junho de 1993. Dispõe sobre a constituição e o regime tributário dos Fundos de Investimento Imobiliário e dá outras providências. Recuperado de: http://www.planalto.g ov.br/ccivil_03/leis/ L8668.htm.

Dempster, M. A. H. (2002). Risk Management: Value at Risk and Beyond. Cambridge: Cambridge University Press.

Elton, E. J., Gruber, M. J., Brown, S. J. \& Goetzmann, W. N. (2012). Moderna Teoria de Carteiras e Análise de Investimentos. Rio de Janeiro: Elsevier.

Elton, E. J., \& Gruber, M. J. (1997). Modern portfolio theory, 1950 to date. Journal of Banking \& Finance, 21(17), 1743-1759.

Fabozzi, F. J., \& Markowitz, H. M. (2011). The Theory and Practice of Investment Management. Hoboken: John Wiley \& Sons. 
Gitman, L. J. (2010). Princípios de Administração Financeira (12 ed.). São Paulo: Pearson.

Jorion, P. (2006). Value at Risk: The New Benchmark for Managing Financial Risk. New York: McGraw-Hill.

FTSE. (2015). FTSE NAREIT US Real Estate Index Series. New York: FTSE Russel.

Kolm, P., Tütüncü, R., \& Fabozzi, F. (2014). 60 Years of portfolio optimization: Practical challenges and current trends. European Journal of Operational Research, 234(2), 356-371.

Markowitz, H. M. (1952). Portfolio Selection. Journal of Finance, 7(1), 77-91.

Markowitz, H. (1959). Portfolio Selection: Efficient Diversification of Investments. New York: John Wiley \& Sons.

Moreira, L. L. (2006). Risco de Mercado: Análise comparativa de métodos de mensuração de risco aplicado ao mercado brasileiro. (Dissertação de mestrado). Universidade de Brasília, Brasília, DF, Brasil.

Nawrocki, D. (1983). A comparison of risk measures when used in a simple portfolio selection heuristic. Journal of Business Finance and Accounting. 10(2), 183-194.

Reilly, F. K., \& Brown, K. C. (2011). Investment Analysis \& Portfolio Management. Mason: South-Western Cengage Learning.

Rockafellar, R. T., \& Uryasev, S. (2002). Conditional value-at-risk for general loss distributions. Journal of Banking \& Finance, 26(7), 1443-1471.

Rockafellar, R. T., \& Uryasev, S. (2000). Optimization of Conditional Value-at-Risk. Journal of Risk, 2(3), 21-41.

Rom, B. M., \& Ferguson, K. W. (1994). Post-modern portfolio theory comes of age. Journal of Investing. 11-17.

Roy, A. D. (1952). Safety first and the holding of assets. Econometrica, 20(3), 431-449.

Strachman, D., \& Bookbinder, R. (2010). Fund of Funds Investing: A Roadmap to Portfolio Diversification. Hoboken: John Wiley \& Sons.

UQBAR. (2015). Anuário Securitização e Financiamento Imobiliário 2015. São Paulo: Uqbar Publicações, 2015.

Uryasev, S. (2010). VaR and CVaR in Risk Management and Optimization. Recuperado de http://www.ise.ufl.edu/ uryasev/files/2011/11/VaR_vs_CVaR_CARISMA_conference_2010.pdf.

Vernimmen, P., Quiry, P., Dallocchio, M., Le Fur, Y., \& Salvi, A. (2014). Corporate Finance: Theory and Practice. Hoboken: Wiley.

Yamai, Y., \& Yoshiba, T. (2005). Value-at-Risk versus expected shortfall: A practical perspective. Journal of Banking \& Finance, 29, 997-1015. 